\title{
Determining the Position and Orientation of In-body Medical Instruments Using Near-Field Magnetic Field Mapping
}

\author{
Vedat Cavlu and Paul Brennan
}

\begin{abstract}
There is a increasing demand for localizing medical implants in-body, such as wireless capsule endoscope (WCE) and Nasogastric tube (NGT). Some studies have been conducted to solve this issue using either permanent magnets, static current sources or RF fields. The permanent magnet fails due to low power, and static current source requires relatively high power source. The RF field source requires high frequencies to get enough precision, which undergoes high attenuation in the body. At low frequency, when the distance between the source and the receiver array is shorter than the wavelength, the far field assumption fails for localization methods. Therefore, we propose a novel method of mapping the magnetic field vector in the near field region, with which wavelength independent localization is done. We did extensive MATLAB and CST Microwave simulations followed by practical experiments. The proposed method has achieved localization accuracy of less than $1 \mathrm{~cm}$ in $\mathrm{Y}-\mathrm{Z}$ plane, $2 \mathrm{~cm}$ in depth (in $\mathrm{X}$-axis) and the maximum orientation error remained $10^{\circ}$ in $3-\mathrm{D}$.
\end{abstract}

Keywords-Nasogastric Tube (NGT), Wireless Capsule Endoscope (WCE), Magnetic Field Mapping (MFM), Near Field, Source Plane (SP), Local Maxima ( $x_{1}$ and $\left.x_{2}\right)$, In-body Medical Instruments (IBMI).

\section{INTRODUCTION}

In-body medical instruments (IBMI) help doctors and other clinicians to diagnose the illnesses of patients or provide the means to feed patients unable to eat normally [1], [2]. For example, the Wirelss capsule endoscope (WCE) is used for examining and inspecting the interior of the gastrointestinal (GI) tract and the Nasogastric (NGT) is used to feed patient with the nutrition that they need while they are unable to eat. Tracking and verifying the position of the NGT can be used to ensure it has been inserted correctly into the stomach, avoiding accidental insertion into the lungs, larynx, trachea or brain [3]. High precision tracking is more important for the WCE, allowing surgeons to determine the location of abnormalities within the GI tract when conducting surgery [4].

There has been a lot of research to overcome the problem of locating these invasive instruments. Yi Wang [12] used received signal strength (RSS) method using two $4 \times 4$ sensor arrays with maximum location error of $100 \mathrm{~mm}$, which is relatively inaccurate. The permanent magnet (PM) technique is used to determine both the position and orientation of the source. The accuracy is high; however, due to the size restriction of the WCE and NGT only small magnets can be used, which is insufficient to provide enough power. Also, presence of any ferromagnetic material in the detecting area could cause failure in accurate localization [13], [14]. Therefore, more focus is given to the electromagnetic field (EMF) localization technique.

The EMF localization method is based on RSS, time of arrival (TOA), time difference of arrival (TDOA) and angle of arrival (AOA). However, in the near field the TOA and TDOA are not achievable as they require very accurate time synchronization of less than $1 n s$ due to the high speed of EM waves $\left(3 \times 10^{8} \mathrm{~m} / \mathrm{s}\right)$. Likewise, the AOA is not practical due to multiple reflections among tissues. Hence, the

V. Cavlu and P. V. Brennan are with the Department of Electronic and Electrical Engineering, Igdir University,Igdir, Turkey and University College London, London WC1E7JE, U.K; (E-mail:vedatcavlu@gmail.com, vedat.cavlu.14@ucl.ac.uk and p.brennan@ucl.ac.uk) studies are focused generally on RSS. Nonetheless, RSS is problematic because tissue-dependent signal attenuation may lead to inaccurate localization. Also, this method requires good knowledge of transmitted power which can change as the resonant frequency of the transmitter will vary depending on the tissue properties. A small inaccurate measurements of one of these parameters could cause high error [1].

To overcome the issue with the localization of IBMI in the near field, a magnetic field based localization technique is proposed. $13.56 \mathrm{MHz}$ signal is applied through solenoid (IBMI) to generate an alternating magnetic field. This frequency was chosen to maximize the signal strength at the receiver while minimizing the attenuation through the tissue [5]. Since the distance between the source and the sensor array $(\leq 20 \mathrm{~cm})$ is much smaller than the wavelength $(22.12$ $\mathrm{m})$, it is in the near field region. In the near field region the plane wave assumption cannot be used as in the far field case because the wavefronts arriving at the neighbouring sensors are spherical. Therefore, the wavefronts are required to be modelled by both direction of arrival and range in which phase difference between neighbouring sensors are needed. It is not possible to calculate the phase difference in this problem due to long wavelength. Thus, the studies in [6]-[11] either need additional pairings or large computational cost owing to the use of algorithms such as MUSIC and ESPRIT, while magnetic field mapping (MFM) does not require additional pairing and computationally cheap.

The novel MFM method presented in this paper does not need time-synchronization and does not get affected by multiple reflections. There is no need to know transmitted power strength or power loss through the tissues. The MFM overcomes issues in the near field localization at relatively low frequency. It achieves higher locating accuracy and low frequency with low computation cost.

The paper is organized as follows; General background is in section II. Simulation Modelling is in section III. Localization and Orientation Methods are explained in section IV. Experimental Set-up and Experimental Results are provided 
in section V. Finally, section VI concludes the paper.

\section{BACKGROUND}

\section{A. Near Field Region}

The space around an antenna is generally divided into nearfield and far-field regions. The near field region is defined based on the transmitter dimension and the the distance to observation point. Suppose the radius of coil as $a$, speed of light $c$, frequency of signal $f$ and distance between the transmitter and receiver is $r$. Then, the near field region is computed as:

$$
\frac{f(r-a)}{c}<<1
$$

\section{B. Magnetic Field Computation}

In the near field region, the electromagnetic field radiates like the static field. Hence, The magnetic field generated by a multi-turn coil at a observation point of $P_{l}$ is calculated by using by using Biot-Savart law. Suppose that the turns of the coil are closely spaced. Then, the coil can be defined as many currents' loop. Based on the superposition principle, the magnetic field observed at point $P_{l}$ is addition of contribution of each turn. As shown in Fig.1, a coil with radius of $a$ is placed on the $\mathrm{X}-\mathrm{Y}$ plane at the origin of the coordinate system. The coil is excited with a sinusoidal current of $I=I_{m} \sin (\omega t+\phi)$, where $I_{m}, \omega, t$ and $\phi$ are peak current, angular frequency, time and the initial phase, respectively.

Now suppose we divide the loop into infinitesimally small size pieces of $I d \vec{l}$ (current's element) as shown Fig.1.

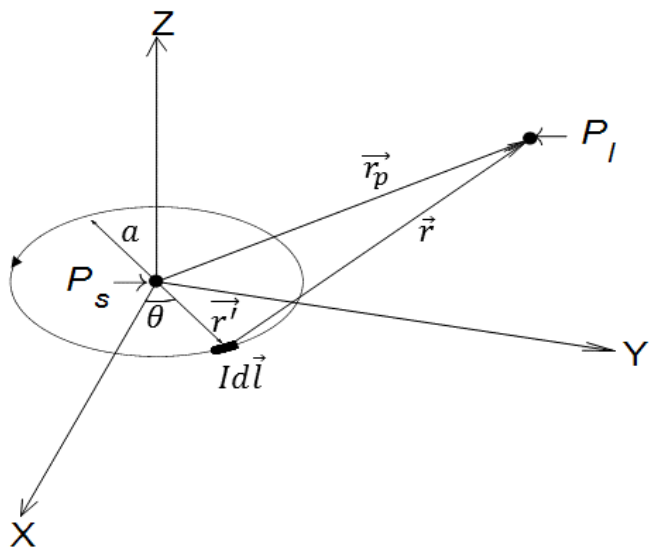

Fig. 1. Magnetic field source (coil) center position $P_{s}\left(x_{s}, y_{s}, z_{s}\right)$ and observation point $P_{l}\left(x_{l}, y_{l}, z_{l}\right)$ position.

Then, the distance from the current's element to the observation point is

$$
r=\sqrt{x_{l}^{2}+y_{l}^{2}+z_{l}^{2}+a^{2}-2 a-2 a y_{l} \sin (\theta)},
$$

and the total incident magnetic field at $P_{l}$ is

$$
B=\frac{\mu_{0} I a}{4 \pi} \int \frac{d \vec{l} \times \vec{r}}{r^{3}} .
$$

Where $x_{l}, y_{l}, z_{l}, a, \mu_{0}$ and $\theta$ are coordinate position of observation point in $\mathrm{X}, \mathrm{Y}$ and $\mathrm{Z}$, the radius of coil, free space magnetic permeability and the angle between the current element and the $\mathrm{X}$-axis, respectively (derivations and details of these equations could be found in [17]). Eq. (3) (Biot-Savart law) is an elliptical equation, as a consequence, it is difficult to solve analytically, so numerical methods are used. Since we are only interested in one component of the field, which is orthogonal to plane of sensors (from now on will be denoted as SP along $\mathrm{X}$-axis), the equation to be used is

$$
B_{x}=\frac{\mu_{0} N I_{m}}{4 \pi} \int_{-\pi}^{\pi} \frac{z_{l} a \cos (\theta) d \theta}{r^{3}} .
$$

Where $B_{x}, N$ are the magnetic field component in $\mathrm{X}$-axis and equal to $B_{\perp}$ in Fig. 2 and coil's number of turns, respectively.

\section{Magnetic Flux Induction Based Faraday's Law}

The source generates an RF alternating magnetic field. The receivers are multi-turn printed circuit board (PCB) rectangular coils. Thus, the system works based on Faraday's law of induction.

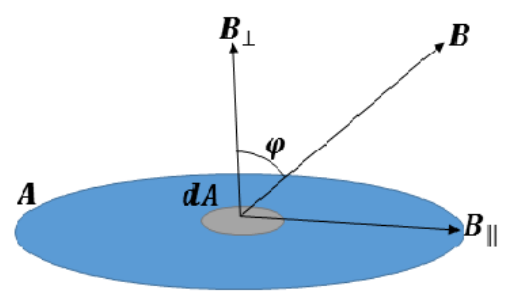

Fig. 2. Magnetic field with angle $\varphi$ to coil surface and the field decomposed to its $B_{\perp}$ and $B_{\|}$components.

Assume we have a coil with area of $A$ (larger circle) as in Fig. 2, the total flux passing through the coil surface is the summation of the field passing through small $d A$ (in grey) areas as given below:

$$
\Phi=\vec{B} \vec{A}=B A \cos (\varphi)=B_{\perp} A=B_{x} A
$$

Therefore, using Faraday's law of induction we obtain induced voltage $\epsilon$ as

$$
\epsilon=-N \frac{d \varphi_{l}}{d t} .
$$

Where $N, B, A, \omega, \varphi$ and $t$ are loop's number of turns, observation point total magnetic field, receiving loop area, angular frequency, angle between incident field its and perpendicular component and time, respectively [16]-[19].

Suppose we have $n \times n$ number of sensors, the induced voltage across $l^{\text {th }}$ sensor could be computed as below by using Eq.(5) and (6):

$$
V_{l}=-N B_{l} A \omega \sin (\omega t)
$$

Therefore, the overall system could be generalized as

$$
V(n \times n)=\left[\begin{array}{ccc}
V_{x 11} & \cdots & V_{x 1 n} \\
\vdots & \ddots & \vdots \\
V_{x n 1} & \cdots & V_{x n n}
\end{array}\right] .
$$

Suppose the source and the 1-D Linear SP is arranged as given in Fig. 3 (a). The number of sensors (on SP) are assumed to be 33 ranging from $S_{1}$ to $S_{33}$, some of which are named in Fig. 3 (a). Then, the results Fig. 3 (b) are obtained using (7). 


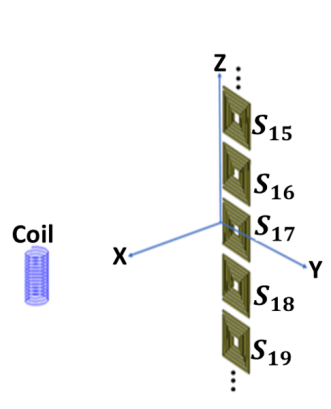

(a)

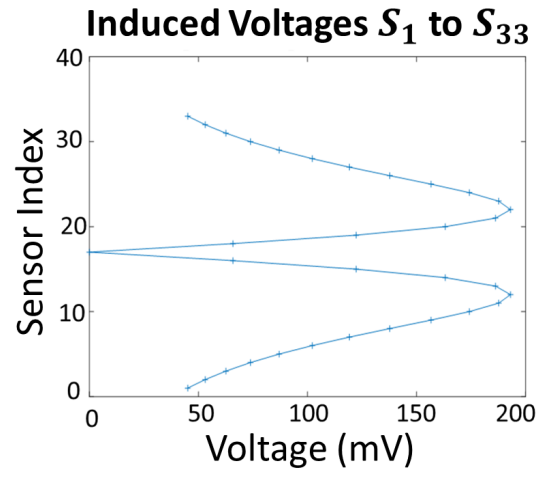

(b)
Fig. 3. The source position relative to 1-D linear sensor array (a) and the field strength at the sensor array from $\mathrm{S}_{1}$ to $\mathrm{S}_{33}(\mathrm{~b})$.

It is clear from Fig.3 (b) that signal strength is weak at $S_{1}$, but it increases almost exponentially as we move from $S_{1}$ to $S_{12}$. Then, it begins a steep decrease from $S_{13}$ to $S_{17}$, at which the strength is zero. The field strength at $S_{18}$ to $S_{33}$ mirror those of $S_{16}$ to $S_{1}$. Hence, this is the crucial information that we will use to deduce the location and orientation of the source as explained in section IV.

\section{Simulation Modelling}

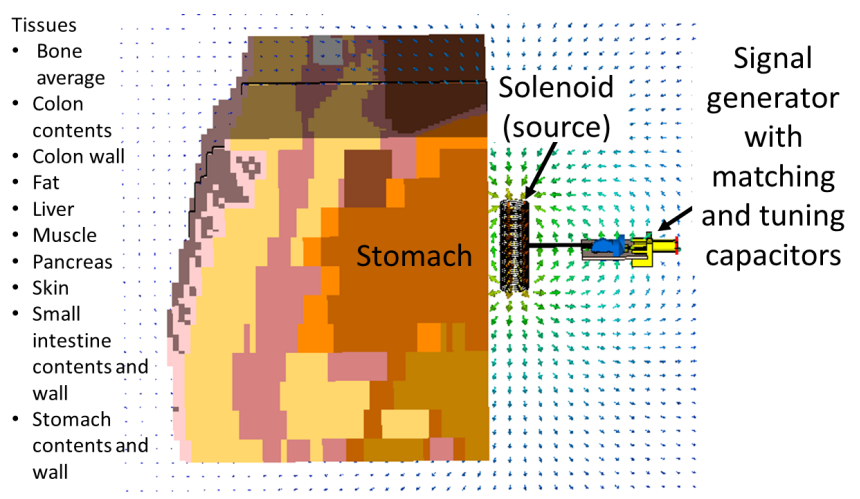

Fig. 4. The magnetic field source (solenoid) and its feeding circuit placed just next to the stomach of CST family voxel ('Donna' as named in CST) and the tissues (colours on voxel represent the different tissues) in the simulation environment listed on the left.

The simulation medium in CST microwave is illustrated in Fig.4. The source is a solenoid of 30 turns with the radius of $3 \mathrm{~mm}$ and length of $20 \mathrm{~mm}$. The source is connected in parallel with a tuning capacitor and in series with a matching capacitor to resonate and keep the load impedance at $50 \Omega$. It is matched to $50 \Omega$ because the highest power can be delivered under impedance match conditions (reader refer to [20] for details).

The simulation was done with the part of human-body starting just below breast to some part of the small intestine. The reason this is done is because the memory required to simulate the entire body exceeded the available computational resources. Two different volumes of voxels were used in the simulations to investigate to effect of vouxel resulution on the computed magnetic field results. The sizes of the two voxel were $85 \times 100 \times 110 \mathrm{~mm}^{3}$ and $110 \times 120 \times 125 \mathrm{~mm}^{3}$. In both cases of voxel simulations, the results were almost the same, and they have negligible effect on the localization system. The Donna is a good human representation (over a homogeneous phantom) as they are built from cross-sectional images obtained using Computer Tomography (CT) and Magnetic Resonance Imaging (MRI) devices. The voxel simulation takes into account the electrical characteristic of tissues individually for the specific frequency of operation. For instance, the blood effect could be different when it's still or flowing, therefore the simulation calculates the effect of blood flow [21]-[23]. To ensure accuracy of the simulation, the minimum mesh step is chosen to be one third of the minimum tissue thickness.

\section{LOCALIZATION AND ORIENTATION METHODS}

In real scenarios, the initial orientation of the source is not known, and as can be seen from Fig.3(a), the peaks are always in parallel with the source orientation. In the proposed method to determine source position and orientation, it is required to have information of the peaks positions and strengths. So, the peaks position is not detected in this case of 1-D array of sensors, it will be applicable only if the orientation of the source is in parallel with the SP array as illustrated in Fig.3(a). Hence, 2-D plane of SP is used Fig. 5 (a).

In 2-D plane, when $\mathrm{P}_{s}$ and $\mathrm{SP}$ are orthogonal to each other as in Fig. 5 (a), the field simulated in CST is given in (b). If Fig.5 (b) is carefully observed, it can be seen that it agrees very well with Fig. 3 (b) because the field between the local maxima is zero. There are two identical, symmetrical field distribution on both sides of the zero line between $\mathrm{x}_{1}$ and $\mathrm{x}_{2}$. The field distribution on both sides of the zero line take an elliptical like shape. The decrease from the maximum point to zero line is steeper than to other sides, which also could be used to give an idea where the source may be located. Under this circumstance, the way of finding the position and orientation of the source was explained in Section II. For different source orientation relative to SP is explained in the following.

\section{A. Determining Angle $\phi$}

If the source is rotated about $V$ axis by $\phi^{\circ}$ in Fig.5 (a), then Eq. (4) will transform into:

$$
B_{x}^{\prime}=c \int_{-N \pi}^{N \pi}\left(\frac{z_{l} a \cos (\theta)}{r^{3}}+\frac{a^{2} \sin (\phi)}{r^{3}}\right) d \theta
$$

The comparison of $B_{x}^{\prime}$ to its first component $(\cos (\theta))$ and second component $(\sin (\phi))$ at different $\phi$ are shown in Fig. 6. It is noticeable from Fig.6 that $B_{x}^{\prime}$ is equal to addition of first and second component on one side of the source center, while on the other side is subtraction of second component from the first. This happens because the first component of the field $(\cos (\theta))$ changes polarity while crossing the center line of the source, while the second component maintains the same sign. Also, local maxima change proportionally with the rotation angle change for $\phi=10^{\circ}$ to $\phi=30^{\circ}$. Hence, this indicates that the local field strengths are related to second component of the field, which depends on the rotation angle of the source. 


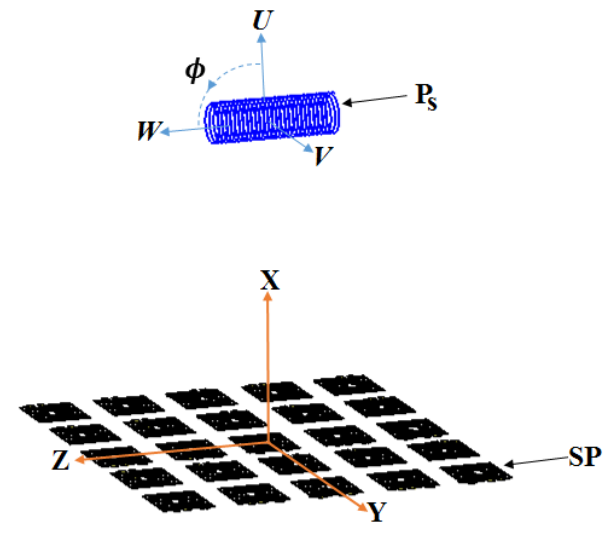

(a)

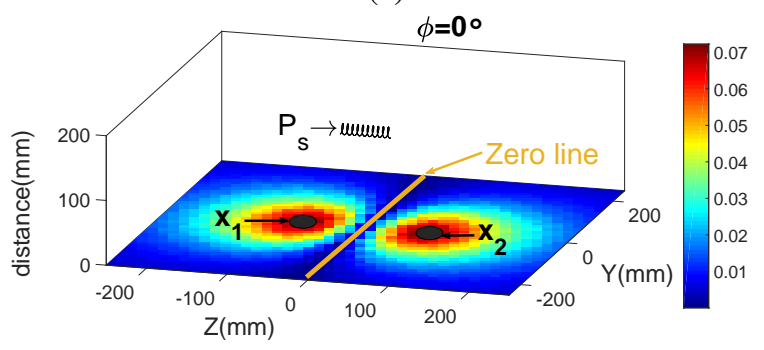

(b)

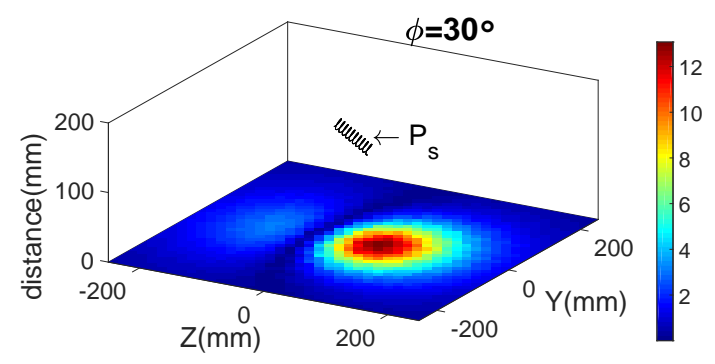

(c)

Fig. 5. Magnetic field source $\mathrm{P}_{s}$ (blue coil) relative to local coordinate system $(U, V, W)$ aligned with $W$ and the receiver coils on the Y-Z plane of the global coordinate system aligned with $\mathrm{X}$ - axis (a), mapped field result for source rotations $0^{\circ}$ (b) and $30^{\circ}$ (c).

Fig. 5 (c) indicates that as the source rotates about $V$ axis, while one maximum field rises, the other one gets weaker. We can use this information to predict the angle relative to SP. From Fig. 6, clearly the change in $\phi$ causes a change in the difference between the two field's maxima $\left(\mathrm{x}_{1}\right.$ and $\mathrm{x}_{2}$ hereon will be described as peaks or maxima interchangeably).

If we plot the change in normalized maximum field differences ( $F$ in Eq.(10)) against $\phi$, Fig. 7 is obtained.

$$
F=\frac{\mathrm{x}_{1}-\mathrm{x}_{2}}{\mathrm{x}_{1}+\mathrm{x}_{2}}
$$

Where $x_{1}$ and $x_{2}$ are local maxima. The graph in Fig. 7 is similar to a typical sine function graph. Hence, if inverse sine function of the normalized field differences are taken $(\phi=$ $\left.\sin (F)^{-1}\right)$, then the rotation angle $(\phi)$ can be predicted. Using this, the source rotation is predicted and the predicted angles versus real angle are illustrated in Fig.8 (a). The Predicted angles' results up to $65^{\circ}$ were obtained with an accuracy of less than $3^{\circ}$ error. However, the results deteriorate when the

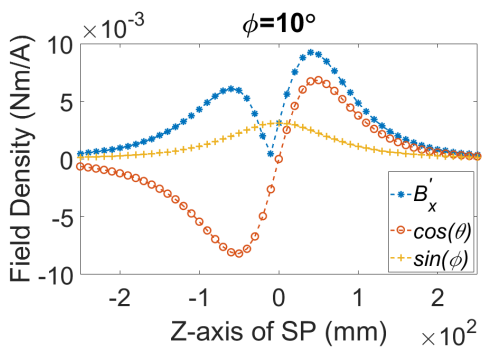

(a)

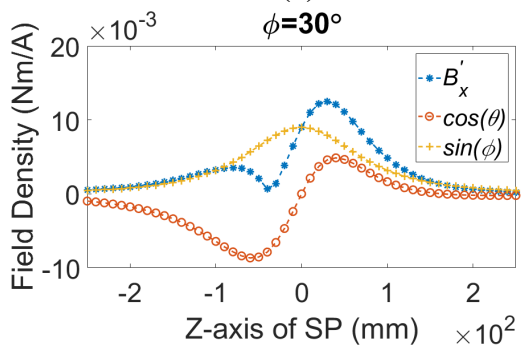

(b)

Fig. 6. Components $\cos (\theta)$ and $\sin (\phi)$ and their absolute total contribution $B_{x}^{\prime}$ field strength at angles of $10^{\circ}$ (a) and $30^{\circ}$ (b).

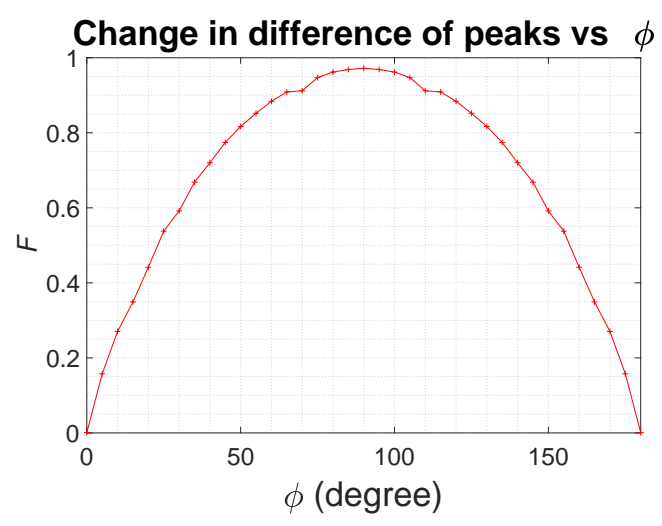

Fig. 7. Change in field difference $F$ versus orientation angle $(\phi)$ of the source towards SP.

angle is at higher degrees depending on the distance between $\mathrm{SP}$ and $\mathrm{P}_{s}$. It is clearly seen from Fig.8 that the angles at $75^{\circ}$ and $90^{\circ}$ result in more inaccuracy. As a consequence, we need to introduce some coefficients to obtain better predicted results. Thus, we empirically used the following equation for the distance between $\mathrm{SP}$ and $\mathrm{P}_{s}$ with $20 \mathrm{~mm}$ increment starting from $100 \mathrm{~mm}$ to $200 \mathrm{~mm}$. At every distance, we have divided predicted ratio $(F)$ to real ratio $(\sin (\phi))$ for every $5^{\circ}$ and taken mean as follows:

$$
C_{l}=\frac{F}{\sin (\phi)}\left\{\mathrm{x}=80+20 l \mathrm{~mm}, \phi=5^{\circ}, 10^{\circ}, \ldots, 90^{\circ}\right.
$$

Where $l=1,2, \ldots, 6$ and $C_{l}$ is a constant matrix coefficient for the distances of X 100 to $200 \mathrm{~mm}$ and $C$ is the mean constant vector coefficient of $C_{l}$ matrix.

$$
\phi=\sin \left(\frac{F}{C}\right)^{-1}
$$

The results in Fig.8 (b) clearly show that the correction coefficients increased the accuracy of prediction of the angles.In 


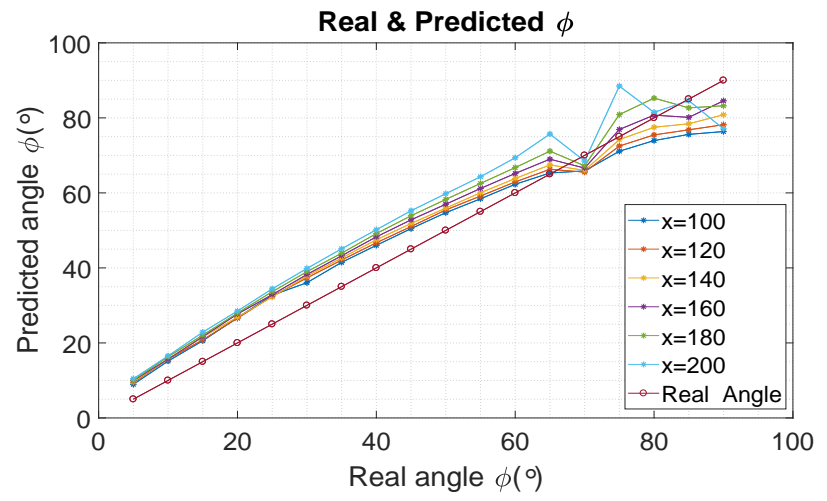

(a)

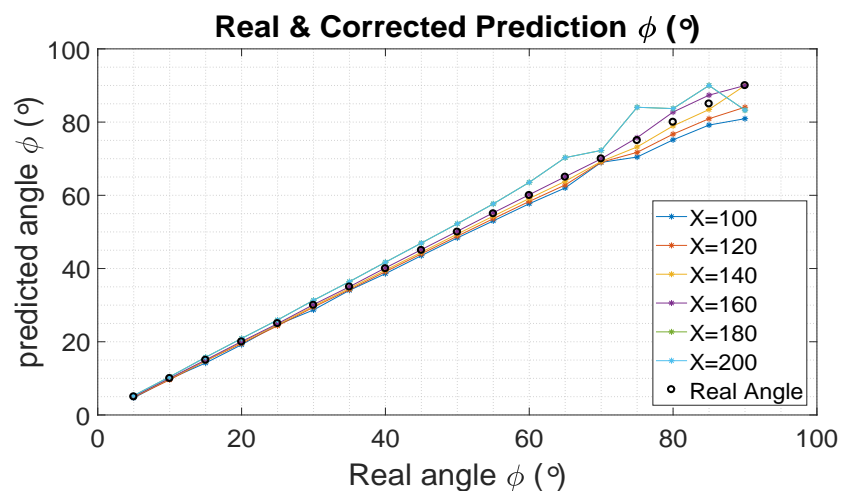

(b)

Fig. 8. Predicted versus real angle (a) and corrected prediction versus real angle (b).

addition to improvement in the accuracy of the angles at the higher degrees, the accuracy of lower angles are improved as well. There is a good agreement between the predicted angles and the real angles. However, the results for angles between $75^{\circ}$ and $90^{\circ}$ have more error relative to lower angles.

\section{B. Source Position on Y-Z Plane}

The relationship between rotation angle and the maxima were explained above. Also, it can be realized from Fig.6 (a) and (b), as the rotation angle increases the increasing peak approaches the peak of second component while decreasing peak moves away from it. Also, notice the second component's peak (shown in Fig.9 as $\mathbf{x}_{\phi}$ ) is always aligned with the source position for any angle. The peaks were obtained in Eq.(9) by adding together two $B_{x}^{\prime}$ components on one side or subtracting from each other on the other side. It was understood from results that the difference between peaks $\mathrm{x}_{3}$ always aligns with the $\mathrm{x}_{\phi}$, and thus this is where the source is positioned.

$$
B_{x}^{\prime}=\mathrm{x}_{3}=\left\|\mathrm{x}_{1}\right\|-\left\|\mathrm{x}_{2}\right\|
$$

But owing to the symmetry of $B_{x}^{\prime}$ around $\mathrm{x}_{1}$, the system could show on both sides $\left(\mathrm{x}_{3}\right.$ and $\left.\mathrm{x}_{4}\right)$, but we know that the source is always between $x_{1}$ and $x_{2}$. Hence, the system can easily detect the position of the source in Y-Z plane.

\section{Third Dimension (Depth) Determination}

In Fig.10, the field mapped for distances of $\mathrm{x}=50 \mathrm{~mm}$ to $\mathrm{x}=200 \mathrm{~mm}$ between the source and SP are given. This

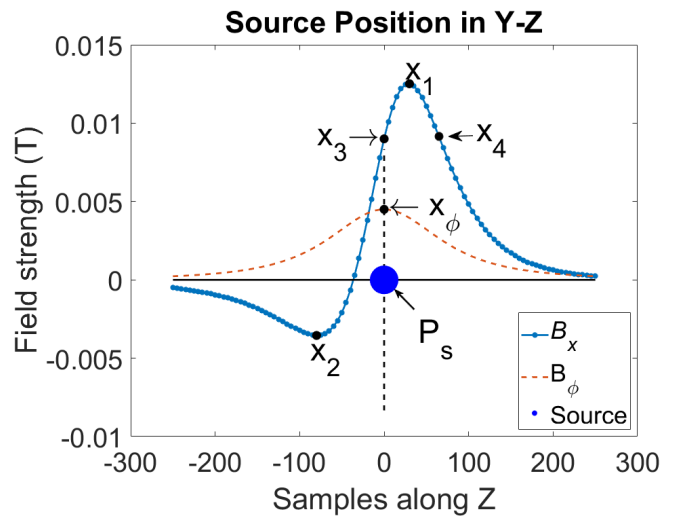

Fig. 9. Predicting the source position at different angles on Y-Z plane

indicates that as the source moves away from the SP ( $\mathrm{x}=$ $50 \mathrm{~mm}$ to $\mathrm{x}=200 \mathrm{~mm}$ ), the field distribution gets wider. It is an indication of the link between the distance from the source to SP and the width of the field distribution. This empirically obtained relationship can be used to estimate the distance in Xaxis direction. The distance can be predicted using the Eq.14.
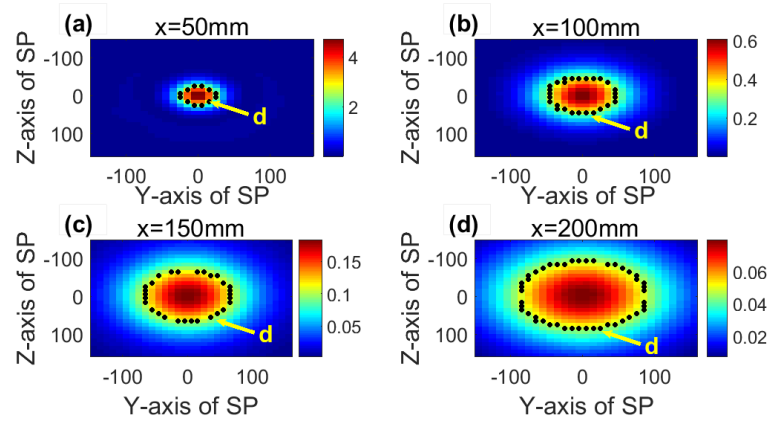

Fig. 10. The field distribution gets wider on the same plane as it moves away from the source (from (a) to (d)) by $\mathrm{x}$ distance.

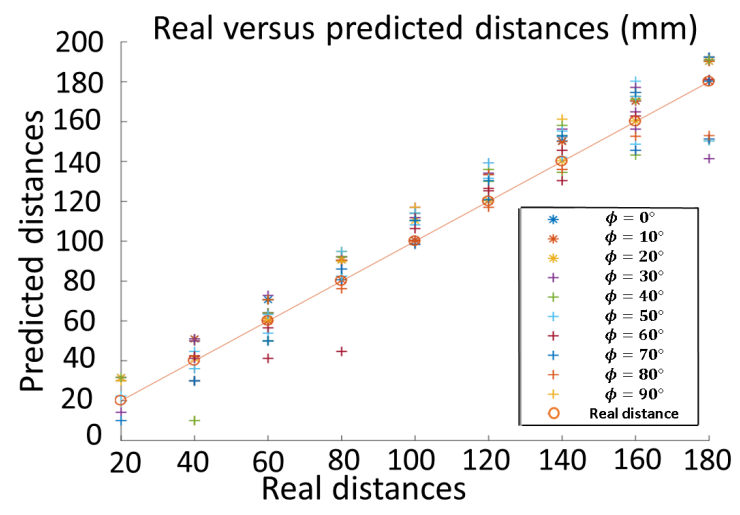

Fig. 11. Real distance versus predicted distance at different angles of rotation.

$$
d=\frac{B_{\max }}{0.56}
$$

The $d$ here represents the half of the maximum field strength measured around $B_{\max }$ (maximum field strength) shown in black circle in Fig.10. We can predict the distance between the SP and the source by using the $d$ circle positions information. 
The depth (x) is proportional to the distance between two farthest $d$ around $B_{\max }$. The Fig.11 displays the predicted versus real distances for different source orientations.

The predicted results are best for all rotation angles between $80 \mathrm{~mm}$ and $140 \mathrm{~mm}$ region. The error is higher (almost $20 \mathrm{~mm}$ for some angles) beyond this region.

\section{EXPERIMENTAL SET-UP AND EXPERIMENTAL RESUltS}

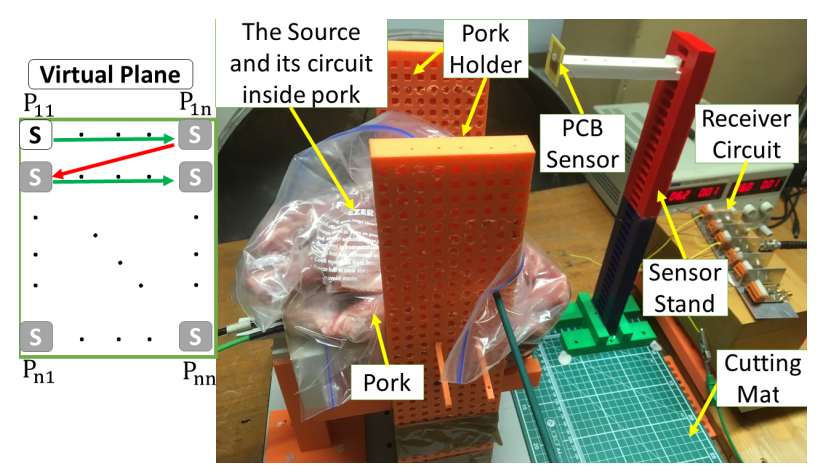

Fig. 12. The feeding circuit \& tilted solenoid (source) on plastic stand and receiver coil on its plastic stand $\&$ its receiver circuit at back.

The experiments were conducted in free space environment and in animal tissues (namely pork) as indicated in Fig.12. The tissues that were used were fresh pork shoulder steaks, belly joint and pork ribs. The tissues were placed around the solenoid (IBMI). The thickness of the tissues between the Source and SP were $9.5 \mathrm{~cm}$ and the layers of each different tissue were distributed equally around the in-body medical instrument. In the experiments, we used a solenoid (source) of 30 turns with radius of $3 \mathrm{~mm}$ and length of $20 \mathrm{~mm}$. Also, the sensor coil with 7 turns and radius of $5 \mathrm{~mm}$ on a PCB board was used as seen in Fig.12.

\section{A. MFM on Virtual Plane}

in order to measure the field on SP, on the receiver side, a single sensor was used instead of a sensor plane $(n \times n$ sensor matrix). As seen in Fig.12, the receiver was put at $125 \mathrm{~mm}$ distance away from the source. To measure the magnetic field at each sensor points on the virtual plane (VP), the receiver is moved virtually to each of the location of the sensors. Firstly, the sensor was placed at point $\mathrm{P}_{11}$ on the VP shown on the left side of Fig.12, and the field at this point is measured \& saved. Then, it was moved to its right by $1 \mathrm{~cm}$ to point $\mathrm{P}_{12}$, and the field was measured \& saved. This process conducted continuously until $\mathrm{P}_{1 n}$ as indicated by green arrow at the top. After, the sensor was placed $1 \mathrm{~cm}\left(\right.$ at $\left.\mathrm{P}_{21}\right)$ below the first point $\mathrm{P}_{11}$ as shown with diagonal red arrow. The field at this point was measured and saved. This procedure was carried out until all the points (from $\mathrm{P}_{11}$ to $\mathrm{P}_{n n}$ ) on the VP were measured and saved. Finally, the results of all sensors on the VP is mapped, and the results Fig.13 (b) were yielded. Then, the results in Fig.13 (c) were achieved by placing the receiver 165 $\mathrm{mm}$ away from IBMI and the same procedures were repeated for measuring the field on SP (VP).

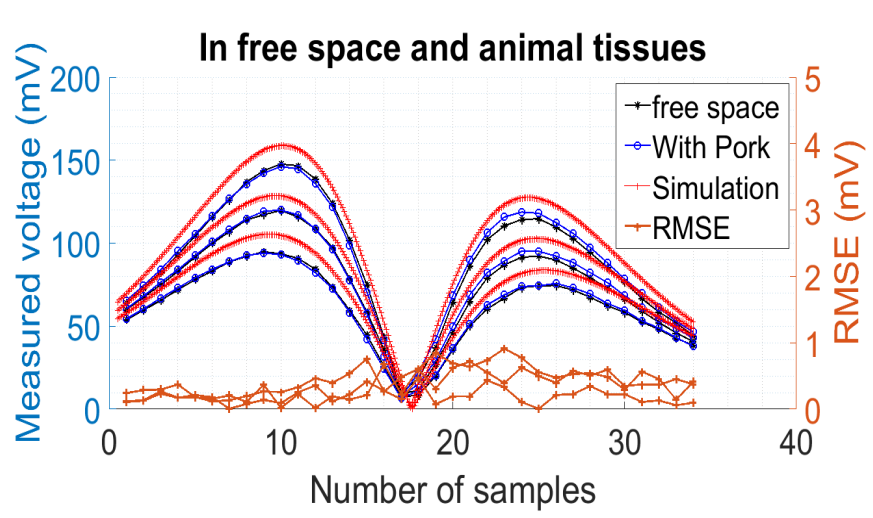

(a)

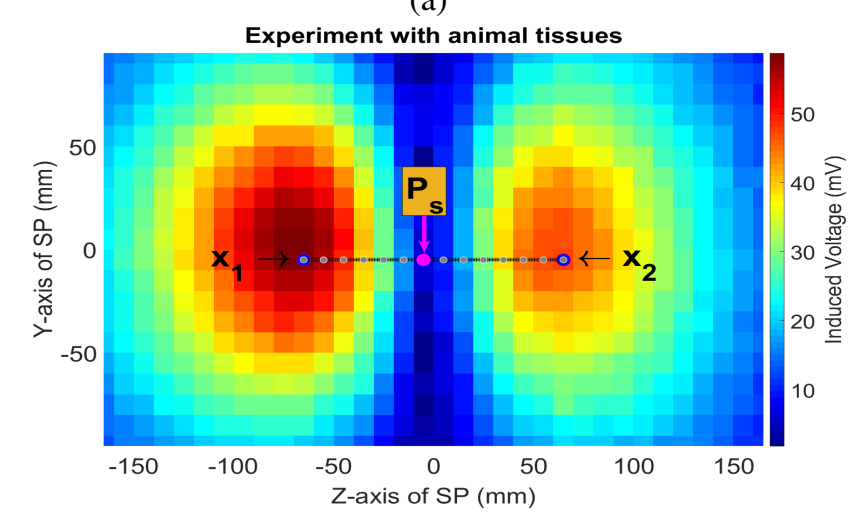

(b)

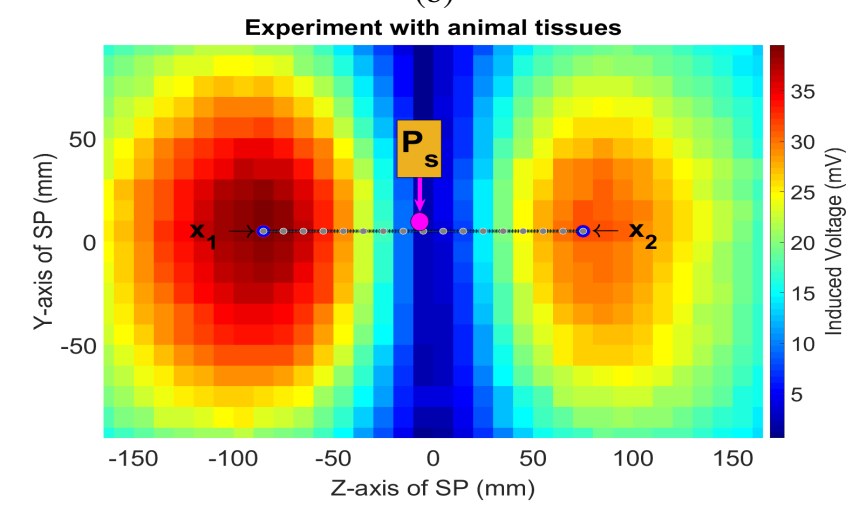

(c)

Fig. 13. Comparison of magnetic field computed in simulation, measured in free space and in animal tissues (a), experimental magnetic field mapping results on 2-D plane at distance of $125 \mathrm{~mm}$ (b), and $165 \mathrm{~mm}$ from $\mathrm{P}_{\mathrm{s}}$ (c).

\section{B. Comparison of Experimental and Simulation Results}

To compare the results of experiments in free space, with the pork tissues and the simulation results, firstly experiments in free space were done along the line in parallel with the source orientation at distances of 120,130 and $140 \mathrm{~mm}$ from the source. Then, the same procedures were conducted while the source was surrounded by the tissues. The results of experiments and simulation are given in Fig.13 (a). Fig.13 (a) shows that the results are very similar. The difference between the experiments in free space and in tissues are calculated by using the root mean square error (RMSE). The RMSE values are indicated on the right axis of Fig.13 (a). The maximum RMSE between the experiments in tissues and in free space is less than $0.92 \mathrm{mV}$. Also, it is clear that the experiment results are in a good agreement with the simulation results. The simulation 
plots were deliberately scaled up for better demonstration of the experiments results. Hence, this results suggests that the effect of the tissues on the field passing through is negligible at the frequency of operation. Furthermore, these results explains that the numerical computations are highly accurate, which means they could be used for localization and orientation calculations.

\section{Magnetic Field Mapping Results on 2-D Plane}

Fig.13 (b) and (c) indicate the experiments results conducted at distances of $125 \mathrm{~mm}$ and $165 \mathrm{~mm}$ from the source to SP, respectively. It could be seen from results that there is a good agreement between the experiment results in Fig.13 (b) and (c) and the simulation results in Fig.5 (b). From Fig.13 (b) and (c), it may be understood that the source is slightly tilted towards the SP in the lower bound (which was $4^{\circ}$ ). The source position is calculated as given in purple circle on the line between two local maxima $\left(\mathrm{x}_{1}\right.$ and $\left.\mathrm{x}_{2}\right)$. The grey circles are the center of the sensors along the line. Also, it is obvious there is a difference between Fig.13 (b) and (c), because in (b) the depth is smaller, the field is more intense in a smaller area. On the contrary, in (c) the field intensity is distributed over a larger area as the depth is bigger, which agrees well with the simulation results Fig.10.

It is understood from the simulation and experiment results that the localization of the source could be performed with an accuracy of less than $1 \mathrm{~cm}$ in the Y-Z axes and $2 \mathrm{~cm}$ in depth (in X-axis). Also, the source orientation in two dimensions is obtained with almost 100 percent accuracy (less than $1^{\circ}$ error). But, the orientation of the source towards the SP is less accurate, which is achieved with a worse case error of less than $10^{\circ}$.

\section{CONCLUSION}

A novel method has been described for near-field location, based on measurement of the magnetic field orientations at a number of sample points. The method was modelled and simulated in Matlab and CST Microwave Studio and compared with experimental results. The results show that there is a good agreement between the simulations and the experiment. It is understood that the source center could be located within $1 \mathrm{~cm}$ Y-Z plane and depth of less then $2 \mathrm{~cm}$. Also the orientation of the source in two dimension could be determined with very high resolution with error of less than $1^{\circ}$. The third orientation could be achieved with maximum error of less than $10^{\circ}$. The results indicates that the method could be used for in body instrument localization with relatively high accuracy.

\section{ACKNOWLEDGMENT}

This research was funded by $\mathrm{PhD}$ scholarship to V. Cavlu by the Turkish Government, Ministry of National Education, Igdir University.

\section{REFERENCES}

[1] T. D. Than, G. Alici, H. Zhou, and W. Li, "A review of localization systems for robotic endoscopic capsules," IEEE Trans. Biomed. Eng., vol. 59, no. 9, pp. 2387-2399, 2012.
[2] Y. Gotoh, A. Nakamura, Y. Miyazaki, and A. I. Model,"Evaluation of Electromagnetic Measuring Technique of Tip Position of Nasogastric Tube Using Evolution Strategy," IEEE Trans. Magnetics, vol. 50, no. 2, pp. 2-5, 2014.

[3] R. Durai, R. Venkatraman, and P. C. H. Ng,"Nasogastric tubes. 2: Risks and guidance on avoiding and dealing with complications." Nursing Times, vol. 105, no. 17. pp. 14-16, 2009.

[4] R. Chandra, A. J. Johansson, and F. Tufvesson, " Localization of an RF source inside the Human body for Wireless Capsule Endoscopy," InProceedings of 8th International Conference on Body Area Networks, Sep. 30, pp. 48-54 2013.

[5] M. Zargham and P. G. Gulak, "Maximum achievable efficiency in nearfield coupled power-transfer systems," IEEE Trans. Biomed. Circuits Syst., vol. 6, no. 3, pp. 228-245, 2012.

[6] J. Yin and T. Chen, "Direction-of-arrival estimation using a sparse representation of array covariance vectors," IEEE Trans. Signal Process., vol. 59, no. 9, pp. 4489-4493, 2011.

[7] Y.D. Huang and M. Barkat, "Near-Field Multiple Source Localization by Passive Sensor Array," IEEE Trans. Antennas Propag., vol. 39, no. 9100566, pp. 968-975, 1991.

[8] N. Yuen and B. Friedlander, "Performance Analysis Of Higher Order ESPRIT For Localization Of Near-field Sources" IEEE Trans. Signal Process., vol. 46, no. 3, pp. 709-719, 1998.

[9] S. Li, W. Liu, D. Zheng, S. Hu, and W. He, "Localization of Near-Field Sources Based on Sparse Signal Reconstruction with Regularization Parameter Selection,” Int. J. Antennas Propag., no. 1260601, p. 7 pages, 2017.

[10] J. Liang and D. Liu,"Passive localization of mixed near-field and farfield sources using two-stage MUSIC algorithm," IEEE Trans. Signal Process., vol. 58, no. 1, pp. 108-120, 2010.

[11] J. Liang and D. Liu, "Passive localization of near-field sources using cumulant," IEEE Sens. J., vol. 9, no. 8, pp. 953-960, 2009.

[12] Y. Wang, R. Fu, Y. Ye, U. Khan, and K. Pahlavan, "Performance bounds for RF positioning of endoscopy camera capsules," 2011 IEEE Radio Wirel. Week, RWW 2011 - 2011 IEEE Top. Conf. Biomed. Wirel. Technol. Networks, Sens. Syst. BioWireleSS 2011, pp. 71-74, 2011.

[13] M. N. Islam and A. J. Fleming, "A novel and compatible sensing coil for a capsule in wireless capsule endoscopy for real time localization," Proc. IEEE Sensors, pp. 1607-1610, 2014.

[14] D. Roetenberg, C. T. M. Baten, and P. H. Veltink, "Estimating body segment orientation by applying inertial and magnetic sensing near ferromagnetic materials," IEEE Trans. Neural Syst. Rehabil. Eng., vol. 15 , no. 1 , pp. 469-471, 2007.

[15] A. Mchutchon, "Electromagnetism Laws and Equations [Textbook]," no. 1, pp. 1-13, 2013.

[16] X. Guo, G. Yan, and W. He, "A position telemetric method for implantable microcapsules in the gastrointestinal tract," Meas. Sci. Technol., vol. 19, no. 4, 2008.

[17] J. Newman, "Physics of the Life Sciences", 1st ed. New York: Springer, 2008.

[18] S. Liao, P. Dourmashkin, and J. W. Belcher, "Chapter 9 - Sources of Magnetic Fields," pp. 1-64, 2004.

[19] X. Guo, C. Wang, and R. Yan, "An electromagnetic localization method for medical micro-devices based on adaptive particle swarm optimization with neighborhood search," J. homepage www.elsevier.com/locate/measurement, vol. 44, no. 5, pp. 852-858, 2011.

[20] B. B. Becciolini, "Impedance Matching Networks Applied to RF Power Transistors,"NXP. Application. Note. pp. 1-16, 2009.

[21] Z. Erlangung, "Detailed Modeling of the Human Body in Motion to Investigate the Electromagnetic Influence of Fields in a Realistic Environment," Technische Universitat Darmstadt, 2018.

[22] B. Models and B. Simulations, "BioMedical Simulations with CST STUDIO SUITE Â®," 2012.

[23] C. Gabriel, S. Gabriel, and E. Corthout, "The dielectric properties of biological tissues : I . Literature survey," Phys . Med . Biol, vol. 41, pp. 2231-2249, 1996. 